\title{
Resonance Superfluidity in a Quantum Degenerate Fermi Gas
}

\author{
M. Holland, ${ }^{1}$ S. J. J. M. F. Kokkelmans, ${ }^{1}$ M. L. Chiofalo, ${ }^{2}$ and R. Walser ${ }^{1}$ \\ ${ }^{1}$ JILA, University of Colorado and National Institute of Standards and Technology, \\ Boulder, Colorado 80309-0440 \\ ${ }^{2}$ INFM and Scuola Normale Superiore, Piazza dei Cavalieri 7, I-56126 Pisa, Italy
}

(Received 22 March 2001; published 31 August 2001)

\begin{abstract}
We consider the superfluid phase transition that arises when a Feshbach resonance pairing occurs in a dilute Fermi gas. We apply our theory to consider a specific resonance in potassium $\left({ }^{40} \mathrm{~K}\right)$, and find that for achievable experimental conditions, the transition to a superfluid phase is possible at the high critical temperature of about $0.5 T_{F}$. Observation of superfluidity in this regime would provide the opportunity to experimentally study the crossover from the superfluid phase of weakly coupled fermions to the Bose-Einstein condensation of strongly bound composite bosons.
\end{abstract}

DOI: 10.1103/PhysRevLett.87.120406

The achievement of Bose-Einstein condensation in atomic vapors [1] has given great impetus to efforts to realize superfluidity in dilute fermionic alkali gases. While conditions of quantum degeneracy have been obtained in potassium $\left({ }^{40} \mathrm{~K}\right)[2]$, the lowest achievable temperatures to date have been limited to around $0.2 T_{F}$ [3]. Although this limit is essentially technical in nature, it appears likely that it will be necessary to utilize a strong pairing mechanism yielding superfluid transition temperatures close to this value.

Even in high- $T_{c}$ superconductors, the typical critical temperatures are of the order of $10^{-2} T_{F}$. In the context of strong-coupling superconductivity there has been much work on constructing minimal models to study the crossover from the seminal Bardeen-Cooper-Schrieffer (BCS) theory [4] for conventional superconductivity to the BoseEinstein condensation of tightly bound pairs, passing through nonperturbative regimes in $T_{c} / T_{F}[5,6]$. In this Letter, we treat explicitly a short range quasibound resonant state by extending the theory given in Refs. [7] to predict the existence of a Feshbach resonance superfluidity in a gas of fermionic potassium atoms. This system has an ultrahigh critical phase transition temperature in close proximity to the Fermi temperature. This is a novel regime for quantum fluids, as illustrated in Fig. 1 where our system and others which exhibit superfluidity or BEC are compared.

The seminal BCS theory [4] of superconductivity applied to a dilute gas considers binary interactions between particles in two distinguishable quantum states, say, $|\uparrow\rangle$ and $|\downarrow\rangle$. For a uniform system, the fermionic field operators may be Fourier expanded in a box with periodic boundary conditions giving wave-vector- $\boldsymbol{k}$ dependent creation and annihilation operators $a_{k \sigma}^{\dagger}$ and $a_{k \sigma}$ for states $|\sigma\rangle$. At low energy, the binary scattering processes are assumed to be completely characterized by the $s$-wave scattering length $a$ in terms of a contact quasipotential $U=4 \pi \hbar^{2} a n / m$, where $n$ is the number density. The Hamiltonian describing such a system is given by
PACS numbers: 03.75.Fi, 05.20.Dd, 05.30.Fk, 67.40.Fd

$$
H=\sum_{k} \epsilon_{k}\left(a_{k \uparrow}^{\dagger} a_{k \uparrow}+a_{k \downarrow}^{\dagger} a_{k \downarrow}\right)+U \sum_{\boldsymbol{k}_{1} \cdots \boldsymbol{k}_{3}} a_{\boldsymbol{k}_{1} \uparrow}^{\dagger} a_{\boldsymbol{k}_{2} \downarrow}^{\dagger} a_{\boldsymbol{k}_{3} \downarrow} a_{\boldsymbol{k}_{4} \uparrow},
$$

where $\epsilon_{k}=\hbar^{2} k^{2} / 2 m$ is the kinetic energy, $m$ is the mass, and the constraint $\boldsymbol{k}_{4}=\boldsymbol{k}_{1}+\boldsymbol{k}_{2}-\boldsymbol{k}_{3}$ gives momentum conservation.

For a negative scattering length, the thermodynamic properties of the gas show a superfluid phase transition at a critical temperature $T_{c}$ which arises due to an instability towards the formation of Cooper pairs. When the gas is dilute, as characterized by the inequality $n|a|^{3} \ll 1$

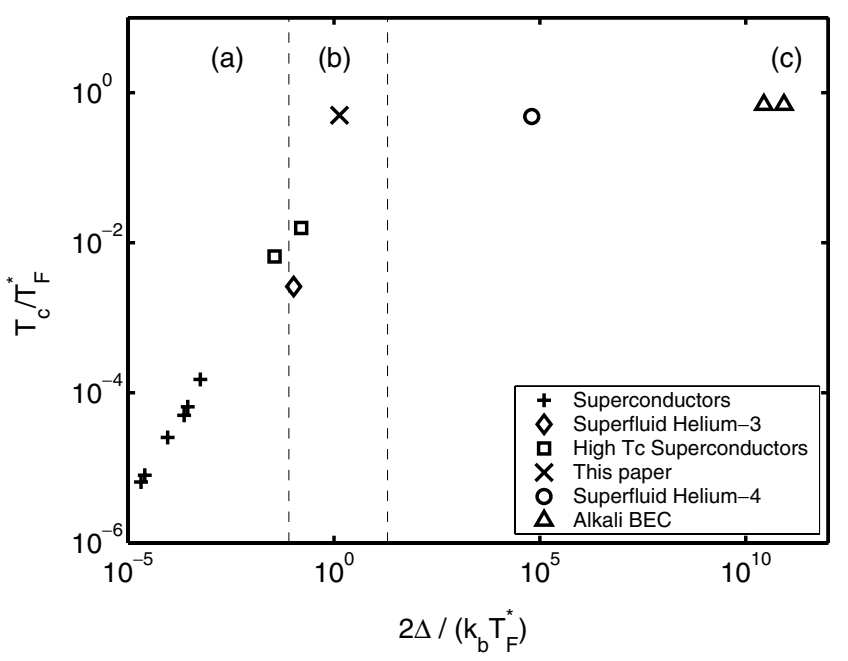

FIG. 1. A log-log plot showing six distinct regimes for quantum fluids. The transition temperature $T_{c}$ is shown as a function of the relevant gap energy $2 \Delta$. Both quantities are normalized by an effective Fermi temperature $T_{F}^{*}$. For the BCS systems in region $(a)$, and the systems in the crossover region $(b), 2 \Delta$ is the energy needed to break up a fermion pair, and $T_{F}^{*}$ is the Fermi energy. For the systems in region $(c)$, which are all strongly bound composite bosons and exhibit BEC phenomenology, $2 \Delta$ is the smallest energy needed to break the composite boson up into two fermions, i.e., ionization to a charged atomic core and an electron, and $T_{F}^{*}$ is the ionic Fermi temperature. 
(or equivalently $k_{F}|a| \ll 1$ where $k_{F}$ is the Fermi wave number), the application of mean-field theory gives a wellknown solution for the ratio of $T_{c}$ to the Fermi temperature $T_{F}$ [5]:

$$
\frac{T_{c}}{T_{F}} \sim \exp \left(-\frac{\pi}{2|a| k_{F}}\right) .
$$

The exact prefactor to the exponential depends on the precise form of the analytic integral approximations made in the derivation. Several papers have pointed out that the presence of a scattering resonance in dilute alkali gases can be used to obtain a very large negative value for the scattering length [8]. This promises the opportunity for the system to enter the high- $T_{c}$ superfluidity regime as the ratio in Eq. (2) approaches unity. However, direct application of the BCS theory close to resonance then becomes speculative due to the potential breakdown of a number of underlying assumptions: (1) Exactly on resonance the theory fails as the scattering length passes through $\pm \infty$ and the Hamiltonian in Eq. (1) cannot be defined. (2) For the mean-field approach to be accurate it is required that there be many particles inside a volume associated with the spatial scale of a Cooper pair. This condition begins to break down as $T_{c}$ approaches $T_{F}$. (3) The theory of the dilute gas is formulated on a perturbation approach based on an expansion in the small parameter $n|a|^{3}$. When this parameter approaches unity the perturbation theory fails to converge. These points show that care should be taken in applying Eq. (2) near the point of resonance where the basis for the conventional mean-field theory is not well founded.

Despite these limitations, on general grounds, one would expect to be able to derive a renormalizable low-energy effective field theory even in close proximity to a resonance. This statement is based on the identification that at relevant densities the range of the interparticle potential is always orders of magnitude smaller than the interparticle spacing. Here we present a theory of superfluidity in a gas of

dilute fermionic atoms which handles correctly the scattering resonance and places the transition temperature to the superfluid state in the experimentally accessible range.

While the scattering length $a$ usually characterizes the range of the interatomic potential for a collision, this is a poor approximation in the vicinity of a scattering resonance. The scattering properties are completely determined by the positions of the bound states in the interaction potentials. In a multichannel system, a bound state may cross the threshold as a function of magnetic field and enter the continuum, resulting in a field-dependent Feshbach scattering resonance [9]. As this occurs, the scattering length becomes strongly dependent on the field, and exactly at threshold it changes sign by passing through $\pm \infty$.

When such resonance processes occur, it is necessary to formulate the Hamiltonian by separating out the resonance state and treating it explicitly. This is motivated by the microscopic identification of two types of scattering contributions: one from the scattering resonance, and one from the background nonresonant processes that includes the contributions from all the other bound states. The nonresonant contributions give rise to a background scattering length $a_{\mathrm{bg}}$ which is a good characterization of the potential range. The corresponding quasipotential in that case is given by $U_{\mathrm{bg}}=4 \pi \hbar^{2} a_{\mathrm{bg}} n / m$. The Feshbach resonance occurs due to a coupling with a molecular state, that is long-lived in comparison with characteristic nonresonant collision time scales. This state is a composite boson which is described by bosonic annihilation operators $b_{\boldsymbol{k}}$. It is parametrized by a detuning energy from threshold, denoted by $2 \nu$, that is dependent on the value of the magnetic field. The coupling strength of $b_{\boldsymbol{k}}$ to the two-particle continuum is well characterized by a single coupling constant $g$, independent of $\boldsymbol{k}$. These considerations imply that the Hamiltonian given in Eq. (1) is not sufficient to account for the important resonance processes and must be extended to incorporate explicitly the coupling between the atomic and molecular gases:

$$
\begin{aligned}
H= & 2 \nu \sum_{\boldsymbol{k}} b_{\boldsymbol{k}}^{\dagger} b_{\boldsymbol{k}}+\sum_{\boldsymbol{k}} \epsilon_{k}\left(a_{\boldsymbol{k} \uparrow}^{\dagger} a_{\boldsymbol{k} \uparrow}+a_{\boldsymbol{k} \downarrow}^{\dagger} a_{\boldsymbol{k} \downarrow}\right)+U_{\mathrm{bg}} \sum_{\boldsymbol{k}_{1} \cdots \boldsymbol{k}_{3}} a_{\boldsymbol{k}_{1} \uparrow}^{\dagger} a_{\boldsymbol{k}_{2} \downarrow}^{\dagger} a_{\boldsymbol{k}_{3} \downarrow} a_{\boldsymbol{k}_{4} \uparrow} \\
& +g \sum_{\boldsymbol{k}, \boldsymbol{q}} b_{\boldsymbol{q}}^{\dagger} a_{\boldsymbol{q} / 2+\boldsymbol{k} \uparrow} a_{\boldsymbol{q} / 2-\boldsymbol{k} \downarrow}+b_{\boldsymbol{q}} a_{\boldsymbol{q} / 2-\boldsymbol{k} \downarrow}^{\dagger} a_{\boldsymbol{q} / 2+\boldsymbol{k} \uparrow}^{\dagger} .
\end{aligned}
$$

Evolution generated by this Hamiltonian conserves the particle number $N=\sum_{k}\left(a_{k \uparrow}^{\dagger} a_{k \uparrow}+a_{k \downarrow}^{\dagger} a_{k \downarrow}\right)+2 \sum_{k} b_{k}^{\dagger} b_{k}$. Note that the Hamiltonian does not contain $a$ explicitly, and that the field dependence of the scattering is completely characterized by the parameters: $g, \nu$, and $U_{\text {bg }}$. The magnitude of $g$ is derived in the following way. We define $\kappa$ as the product of the magnetic field width of the resonance and the magnetic moment difference of the Feshbach state and the continuum state. For large values of $\nu$, the boson field $b_{k}$ can be adiabatically eliminated from the theory, and then $g=\sqrt{\kappa U_{\mathrm{bg}}}$ is required in order for the scattering properties to have the correct dependence on magnetic field [10].
The essential point is that this Hamiltonian, founded on the microscopic basis of resonance scattering, is well behaved at all detunings $\nu$; even for the pathological case of exact resonance. The diluteness criterion is now given by constraints which require both the potential range and the spatial extent of the Feshbach resonance state to be much smaller than the interparticle spacing (e.g., $n\left|a_{\mathrm{bg}}\right|^{3} \ll 1$ ).

We apply this Hamiltonian to derive the self-consistent mean fields for given thermodynamic constraints by formulating a Hartree-Fock-Bogoliubov theory [11]. The mean fields present include the fermion number $f=\sum_{k}\left\langle a_{k \uparrow}^{\dagger} a_{k \uparrow}\right\rangle$, the molecule field $\phi_{m}=\left\langle b_{k=0}\right\rangle$ taken to be a classical 
field, and the pairing field $p=\sum_{k}\left\langle a_{k \uparrow} a_{-k \downarrow}\right\rangle$ [12]. It is well known that such a theory must be renormalized in order to remove the ultraviolet divergence which arises from the incorporation of second-order vacuum contributions. This implies replacing the physical parameters in the Hamiltonian, $U, g$, and $\nu$, by renormalized values so that observables are independent of a high momentum cutoff used in the formulation of the effective field theory [13]. In order to diagonalize the Hamiltonian, we construct Bogoliubov quasiparticles according to the general canonical transformation [14]

$$
\left(\begin{array}{c}
\alpha_{k \uparrow} \\
\alpha_{-k \downarrow}^{\dagger}
\end{array}\right)=\left(\begin{array}{cc}
\cos \theta & -e^{i \gamma} \sin \theta \\
e^{i \gamma} \sin \theta & \cos \theta
\end{array}\right)\left(\begin{array}{c}
a_{k \uparrow} \\
a_{-k \downarrow}^{\dagger}
\end{array}\right) .
$$

Given single particle energies, $U_{k}=\epsilon_{k}-\mu+U f$, where $\mu$ is the chemical potential, and the gap parameter in the quasiparticle spectrum $\Delta=U p-g \phi_{m}$, the two transformation angles are specified as $\tan (2 \theta)=|\Delta| / U_{k}$ and $\phi_{m}=\left|\phi_{m}\right| \exp (i \gamma)$. The corresponding quasiparticle spectrum is $E_{k}=\sqrt{U_{k}^{2}+\Delta^{2}}$. Dropping terms of higher order than quadratic in the fermion operators gives the resulting many-body Hamiltonian

$$
\begin{aligned}
H-\mu N= & 2(\nu-\mu)\left|\phi_{m}\right|^{2} \\
& +\sum_{k}\left[U_{k}+E_{k}\left(\alpha_{k \uparrow}^{\dagger} \alpha_{k \uparrow}+\alpha_{k \downarrow}^{\dagger} \alpha_{k \downarrow}-1\right)\right],
\end{aligned}
$$

which is now in diagonal form.

The next task is to calculate the thermodynamic solutions. Equilibrium populations for the quasiparticles are given by the Fermi-Dirac distribution. The fermion number and pairing field are not only inputs to the Hamiltonian, but also determine the quasiparticle spectrum. Therefore, they must be self-consistent with the values derived by summing the relevant equilibrium density matrix elements over all wave numbers. In practice, at a given temperature, chemical potential, and molecule number $\phi_{m}$, this requires an iterative method to locate self-consistent values for $f$ and $p$. The value of $\phi_{m}$ is calculated by minimizing the grand potential $\Phi_{G}=-k_{b} T \ln \Xi$ at fixed temperature and chemical potential, with $k_{b}$ denoting Boltzmann's constant. The partition function $\Xi=\operatorname{Tr}\{\exp [-(H-\mu N) /$ $\left.\left.k_{b} T\right]\right\}$ is found from Eq. (5). This procedure is mathematically equivalent to minimizing the Helmholtz free energy at fixed temperature and density and corresponds uniquely to the maximum entropy solution. This solution has an associated particle number, $\langle N\rangle=-\partial \Phi_{G} / \partial \mu$, taken at constant temperature and volume, which must match the actual particle density of the gas, so that the final step is to adjust the chemical potential until this condition is satisfied. The whole procedure is repeated over a range of temperatures to determine the locus of thermodynamic equilibrium points. For large positive detunings, where the molecule field could be eliminated from the theory entirely, regular BCS theory emerges. For this case, when the scattering length $a$ is negative the behavior of the critical temperature on $1 / a$ is given by the usual exponential law [5].
As an example of the application of this theory, we study the experimentally relevant system of fermionic ${ }^{40} \mathrm{~K}$ atoms equally distributed between the two hyperfine states which have the lowest internal energy in the presence of a magnetic field. The values of our interaction parameters $a_{\mathrm{bg}}=176 a_{0}$ and $\kappa / k_{b}=657 \mu \mathrm{K}$ are obtained from [15]. We fix the total density to be $n=10^{14} \mathrm{~cm}^{-3}$, a typical experimental value expected for this quantum degenerate gas in an optical trap. We set the detuning to be $\nu=+E_{F}$ so that the quasibound state is detuned slightly above the atomic resonance. For a temperature above $T_{c}$, the grand potential surface is shaped like a bowl, and the value of $\phi_{m}$ which minimizes the grand potential is $\phi_{m}=0$, associated with the self-consistent solution $p=0$. For $T<T_{c}$, the grand potential surface is shaped like a Mexican hat, and its minimum is given by a $\phi_{m}$ with nonzero amplitude and an undetermined phase. The superfluid phase transition therefore leads to a spontaneously broken symmetry. The value of $T_{c}$ can be clearly found from Figs. 2 and 3, where we show the chemical potential, the molecular density, and the gap as a function of temperature. We find for our parameter set for ${ }^{40} \mathrm{~K}$ and almost zero detuning a remarkably high value for the critical temperature $T_{c} \approx 0.5 T_{F}$, i.e., $T_{c} \approx 0.6 \mu \mathrm{K}$. Furthermore, we find a weak dependence of $T_{c} \approx 0.5 T_{F}$ on the density, so that the value of $T_{c}$ has more or less the same density behavior as $T_{F}$. When we increase the detuning to $\nu=+17.6 E_{F}$ (this corresponds to a magnetic field detuning of $0.5 \mathrm{G}$ away from the Feshbach resonance), the value of $T_{c}$ drops to approximately $0.25 T_{F}$.

The system of ${ }^{40} \mathrm{~K}$ atoms, equally distributed among the two lowest hyperfine states, is a good candidate for demonstrating the superfluid phase transition. It not only exhibits a Feshbach resonance, but also the inelastic binary collision events are energetically forbidden. Three-body interactions are highly suppressed, since the asymptotic three-body wave function should consist of a product

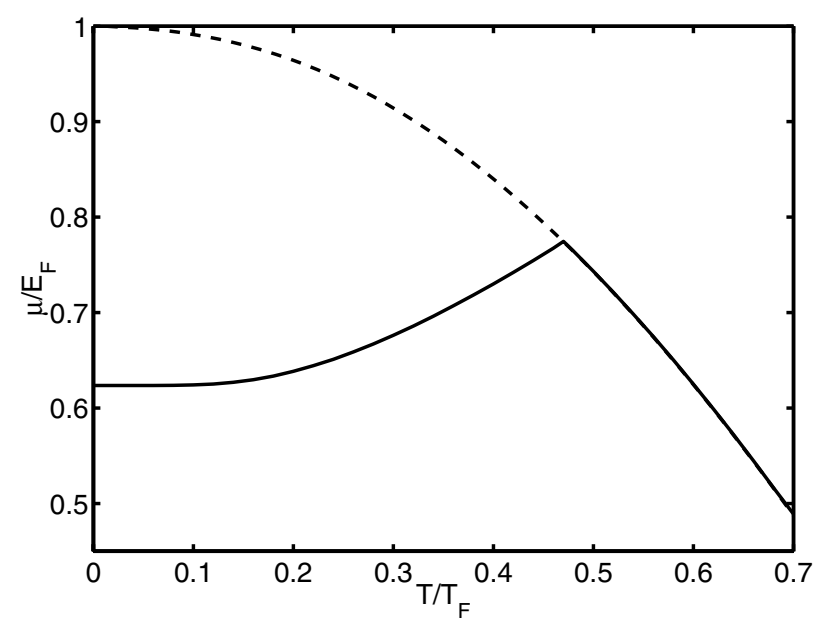

FIG. 2. Chemical potential as a function of temperature for the system of resonance pairing (solid line). The second-order phase transition occurs at $T_{c} \approx 0.5 T_{F}$ where a clear cusp is visible. The dashed line shows the chemical potential of a noninteracting Fermi gas. 


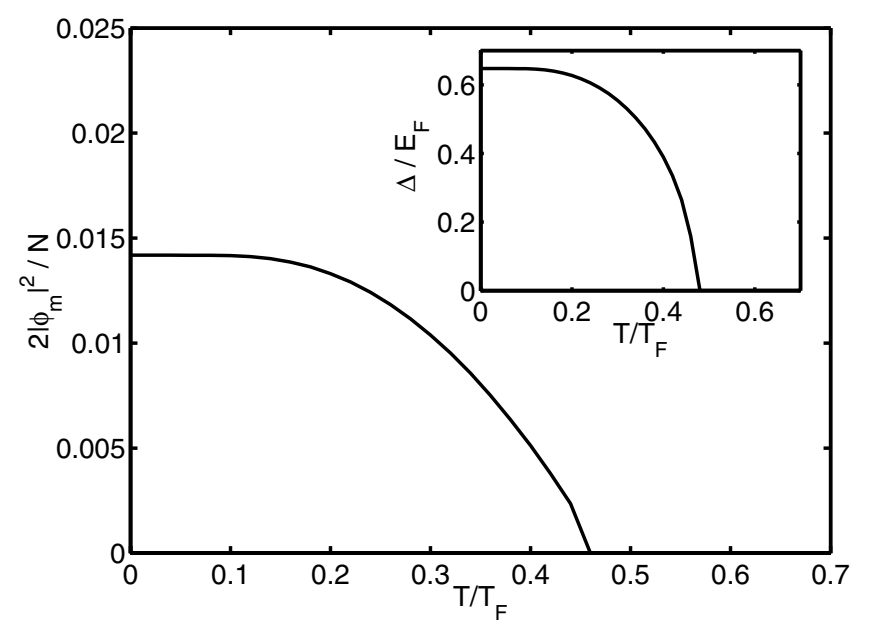

FIG. 3. The temperature at the phase transition is also visible from the amplitude of the molecular field. This amplitude is nonzero only when the broken symmetry exists in the region $T<T_{c}$. For $T=0$, the molecules form a Bose condensed fraction of $1.5 \%$ of the total gas sample. The inset shows the behavior of the gap $\Delta=U p-g \phi_{m}$. The critical temperature $T_{c}$ can be related to the value of the gap at $T=0$. For comparison, in superconductors the analogous gap is simply the binding energy of a fermion pair.

of three $s$-wave two-body scattering wave functions. In a three-body interaction, two particles are always in the same initial hyperfine state, and therefore the corresponding $s$-wave state is forbidden. The only three-body relaxation could come from asymptotic $p$ waves, but these have very little contribution at the low temperatures considered. Although the detailed three-body collision problem is an intricate one, this asymptotic statistical effect should lead to a large suppression of the vibrational relaxation of quasibound molecules.

Current experimental techniques for ultracold gases do not produce samples which are spatially uniform. An optical dipole trap may be needed to confine the high field seeking atoms, and the conditions for the superfluid phase transition would be satisfied first in the trap center where the density is highest. The presence of the quasibound molecules may be a very useful aspect allowing direct observation of the phase transition through imaging the molecular field.

In conclusion, we have shown that resonance pairing in an alkali gas yields a quantum fluid that can undergo a superfluid phase transition at a temperature comparable to the Fermi temperature. This extraordinary property places this system in a regime which lies in between BCS-like superconductors, and bosonic systems which may undergo BEC. Since the transition temperature is larger than the lowest temperatures already achieved in a degenerate Fermi gas, it should be possible to study this new type of quantum matter and to quantitatively compare with our predictions.

We thank J. Cooper, E. Cornell, D. Jin, C. Wieman, and B. DeMarco for very stimulating discussions. Support is acknowledged for M. H. from the National Science Foundation, for S. K. from the U.S. Department of Energy, Of- fice of Basic Energy Sciences via the Chemical Sciences, Geosciences and Biosciences Division, for M. C. from the INFM and SNS, Pisa (Italy), and for R. W. from the Austrian Academy of Sciences.

Note added.-A similar treatment has recently been proposed by Timmermans et al. [16].

[1] M. H. Anderson, J. R. Ensher, M. R. Matthews, C. E. Wieman, and E. A. Cornell, Science 269, 198 (1995); K. B. Davis, M.-O. Mewes, M. R. Andrews, N. J. van Druten, D. S. Durfee, D. M. Kurn, and W. Ketterle, Phys. Rev. Lett. 75, 3969 (1995); C. C. Bradley, C. A. Sackett, J. J. Tollett, and R. G. Hulet, Phys. Rev. Lett. 75, 1687 (1995); 79, 1170(E) (1997).

[2] B. DeMarco and D. S. Jin, Science 285, 1703 (1999).

[3] M. J. Holland, B. DeMarco, and D. S. Jin, Phys. Rev. A 61, 053610 (2000); B. DeMarco, S. B. Papp, and D. S. Jin, Phys. Rev. Lett. 86, 5409 (2001).

[4] J. Bardeen, L. N. Cooper, and J. R. Schrieffer, Phys. Rev. 108, 1175 (1957); J. R. Schrieffer, Theory of Superconductivity (Perseus Books, Reading, MA, 1999).

[5] A. G. Leggett, J. Phys. (Paris) C7, 19 (1980); M. Houbiers and H. T. C. Stoof, Phys. Rev. A 59, 1556-1561 (1999); G. Bruun et al., Eur. Phys. J. D 7, 433-439 (1999); H. Heiselberg, C. J. Pethick, H. Smith, and L. Viverit, Phys. Rev. Lett. 85, 2418 (2000).

[6] See M. Randeria, in Bose-Einstein Condensation, edited by A. Griffin, D. W. Snoke, and S. Stringari (Cambridge University Press, Cambridge, 1995), and references therein.

[7] J. Ranninger and S. Robaszkiewicz, Physica (Amsterdam) 53B, 468 (1985); R. Friedberg and T. D. Lee, Phys. Rev. B 40, 6745 (1989).

[8] H. T. C. Stoof, M. Houbiers, C. A. Sackett, and R. G. Hulet, Phys. Rev. Lett. 76, 10 (1996); R. Combescot, Phys. Rev. Lett. 83, 3766 (1999).

[9] H. Feshbach, Ann. Phys. (N.Y.) 5, 357 (1958); E. Tiesinga, B. J. Verhaar, and H. T. C. Stoof, Phys. Rev. A 47, 4114 (1993); S. Inouye, M. R. Andrews, J. Stenger, H.-J. Miesner, D. M. Stamper-Kurn, and W. Ketterle, Nature (London) 392, 151 (1998).

[10] This expression for $g$ is chosen so that $a$ obeys the correct field dependence. For further discussion see E. Timmermans et al., Phys. Rep. 315, 199 (1999).

[11] An analogous field theory is derived for a bosonic model in M. Holland, J. Park, and R. Walser, Phys. Rev. Lett. 86, 1915 (2001).

[12] A magnetization field $\sum_{k}\left\langle a_{k \uparrow}^{\dagger} a_{k \downarrow}\right\rangle$ is also included in our formulation. However, we drop this term in our discussion since it is identically zero in the spin-symmetric case considered here. Inclusion gives a slightly more general treatment, and requires the addition of a spin rotation following the transformation to Bogoliubov quasiparticles.

[13] S. J. J. M.F. Kokkelmans, J. Milstein, M. Chiofalo, R. Walser, and M. Holland (to be published).

[14] N. N. Bogoliubov, Nuovo Cimento 7, 6 (1958); 7, 794 (1958); J. Valatin, Nuovo Cimento 7, 843 (1958).

[15] John L. Bohn, Phys. Rev. A 61, 053409 (2000).

[16] E. Timmermans, K. Furuya, P. W. Milonni, and A. K. Kerman, Phys. Lett. A 285, 228 (2001). 